\title{
Versorgungsforschung: Das Gesundheitssystem weiterentwickeln
}

\author{
Dr. Christine Blome, Professor Wiebke Ludwig-Peitsch und Dr. Rachel Sommer geben uns eine Einschätzung zu den \\ Hot Topics in der Versorgungsforschung 2020
}

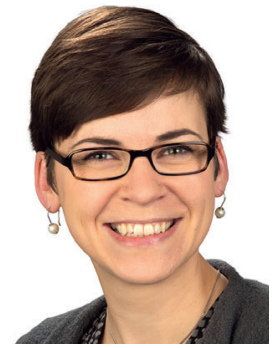

Dr. Christine Blome

Leiterin der

Forschungsgruppe

Lebensqualitäts-

forschung und

Patientennutzen,

IVDP, UKE Hamburg.

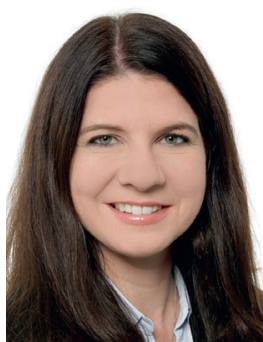

Prof. Dr. Wiebke Ludwig-Peitsch

Chefärztin der Klinik für Dermatologie und Phlebologie, Vivantes Klinikum im Friedrichshain, Berlin.

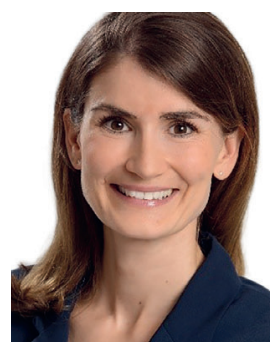

Dr. Rachel Sommer Leiterin der Forschungsgruppe Personenzentrierte Versorgungsforschung, IVDP, UKE Hamburg.
«Die Qualitätsmaßstäbe für die Ergebnismessung aus Patientensicht werden strenger. Das gilt auch bereits für die Entwicklung von Patient Reported Outcomes. Zunehmend wird erwartet, dass bei Letzteren entsprechend der Guidelines, z. B. der FDA-Roadmap, vorgegangen wird.

Damit zusammenhängend wird stärker auf klare Definitionen, zum Beispiel der gesundheitsbezogenen Lebensqualität, geachtet. Das heißt, dass der Entwicklung ein konzeptionelles Modell zugrunde liegt, das darstellt, aus welchen Aspekten sich z. B. «Lebensqualität» zusammensetzt. Hieraus kann dann abgeleitet werden, was genau gemessen werden soll.

Patient Reported Outcome Measures werden außerdem immer häufiger in der Praxis eingesetzt. Die Daten dienen dabei als Basis für patientenorientierte Versorgung, für partizipative Entscheidungsfindung, aber auch für die Qualitätssicherung.

Die Digitalisierung kann die Nutzung in der Praxis noch weiter voranbringen; immer weniger Daten werden auf Papier gespeichert. Informationen werden zunehmend von den Patienten selbst digital erhoben, sodass diese in der Praxis/Klinik eingesehen und besprochen werden können und somit das Gespräch mit dem Patienten effizient gestaltet werden kann. Mit entsprechenden Apps könnten die Patienten dann wiederum stärker in das Management der eigenen Erkrankung eingebunden bzw. hierzu befähigt werden.»
«Spannend finde ich die Themen Patient Reported Outcomes und Patienten-Benefit. Wir haben z.Z. ein Projekt, das sich mit Patientenpräferenzen für die Behandlung des fortgeschrittenen malignen Melanoms beschäftigt. Bei der Auswahl der Therapie ist es nicht nur bedeutsam, welches Medikament wie sicher und effektiv ist.

Entscheidend ist auch, was sich der Patient von seiner Therapie wünscht.

Ein weiteres wichtiges Thema ist die Entwicklung von Registern. Ein gutes Beispiel ist das PsoBest Register, in das mittlerweile über 12000 Patienten eingeschlossen sind. Register müssen auch für andere Dermatosen weiter vorangetrieben werden, um Krankheitsverläufe systematisch zu dokumentieren und den Erfolg der einzelnen Therapien im Alltag einschätzen zu können.

Zu vielen Hauterkrankungen liegen Leitlinien vor. Die hierin empfohlenen Therapien kommen allerdings nicht allen Patienten zugute. Unterversorgung trotz Verfügbarkeit von hocheffektiven Therapien ist ein großes Problem. Sie basiert teilweise darauf, dass bestimmte Medikamente in manchen Bundesländern nicht extrabudgetär verordnet werden können. Es ist auch eine politische Aufgabe, die Ärzte in die Situation zu bringen, ohne Angst vor Regress leitliniengerecht verordnen zu können.

Ein spannendes Thema ist auch die Telemedizin. Es kristallisiert sich gerade heraus, für welche Krankheitsbilder und Situationen Telemedizin geeignet ist und wie sich der damit verbundene Aufwand abbilden lässt.»
«Die Bestrebungen auf nationaler und internationaler Ebene, die Patienten- und Nutzerorientierung im Gesundheitswesen voranzutreiben, nehmen seit Jahren zu. Hierzu gehören zum einen die Ausrichtung der Versorgung an den Bedürfnissen von Betroffenen und ihren Angehörigen. Wichtig ist auch die partizipative Entscheidungsfindung, ebenso wie partizipative Forschung unter Beteiligung von Betroffenen.

Die Versorgungsforschung kann hier aufgrund ihrer vielfältigen Erfahrungen aus wissenschaftlichen Projekten, vielseitigen methodischen Ansätzen und ihrer Interprofessionalität entscheidend dazu beitragen, die Realisierung eines personenzentrierten Gesundheitssystems zu befördern. Gemeinsam mit Betroffenen sowie Behandlerinnen und Behandlern entwickelte Interventionen und methodisch hochwertige Evaluationen können die notwendige Evidenz für diese Weiterentwicklung des Gesundheitssystems generieren und gesundheitspolitische Entscheidungen zu mehr Patientenzentrierung entscheidend vorbereiten. Auch in der Dermatologie gibt es dazu sehr positive Entwicklungen. Schauen Sie auf das vom Bundesministerium für Gesundheit geförderte Projekt zur Entstigmatisierung von Menschen mit sichtbaren chronischen Hauterkrankungen, kurzECHT. Dazu werden derzeit durch ein multidisziplinäres Team verschiedene Interventionen für Betroffene und Nichtbetroffene entwickelt und anschließend getestet, wissenschaftlich evaluiert und langfristig implementiert. Zentral hierbei: die Lebenswelt Betroffener wird einbezogen und Betroffene und Nichtbetroffene begegnen sich.» 